Journal of Mathematics and Statistics 8 (3): 373-376, 2012

ISSN 1549-3644

(C) 2012 Science Publications

\title{
Number of Non-Unique Minors (of Various Orders) and Elements in the Calculation of General Determinants
}

\author{
${ }^{1}$ Patrick Marchisella and ${ }^{2}$ Gurudeo Anand Tularam \\ ${ }^{1}$ Environmental Futures Research Centre, \\ Griffith University, Brisbane Australia \\ ${ }^{2}$ Department of Mathematics and Statistics, \\ Science Environment Engineering and Technology (ENV), \\ Griffith University, Brisbane Australia
}

\begin{abstract}
Problem statement: Many distinct properties of determinants have been studied and are known, yet a considerable number of properties still need further examination. This study investigates the number of minors (of various orders) and elements of a matrix A contained in the expansion of the general determinant of $\mathrm{A}$, irrespective of the independence, principality and distinctness of such minors and elements. Approach: A mathematical proof based approach is taken. Minors of all orders and elements in the calculations of general determinants of matrices of sizes $2 \times 2,3 \times 3,4 \times 4$ and $5 \times 5$ respectively, are considered. Results: Two general expressions involving factorial terms are found: the first being equivalent to the number of minors of various orders found in the analysis of the considered matrices (mentioned above) and the second being equivalent to the number of elements found in the same analysis. Proofs are then presented showing that the expressions hold in the general case of a matrix of size $n \times n$. Conclusion: The results of this study present, with proof, expressions for the total number of minors (of various orders) and elements, respectively, in the general determinant of a matrix of size $n \times n$, irrespective of the independence, principality and distinctness of such minors and elements. Scope for further theoretical study, with applications in applied mathematics and the physical and computer sciences is also indicated.
\end{abstract}

Key words: Factorial terms, mathematical proof, theoretical studies, linear algebra

\section{INTRODUCTION}

The calculation of minors and determinants are crucial in many areas of mathematics, particularly in the teaching of linear algebra. In fact, interest in knowing the number of minors in the expansion of a general determinant dates back to 1928 , when Stouffer determined an expression for the general determinant of a matrix in terms of its principal minors (Stouffer, 1928). In the same paper, Stouffer claimed that an expression for the number of independent principal minors in a matrix was known to MacMahon and later simplified by Muir.

Aitken studied the number of distinct terms in the expansion of symmetric and skew determinants. Later in 1960, Metzler revised Muir's work titled 'The Theory of Determinants in the Historical Order of Development' (Muir, 2008), in which he derived the number of additive terms in the expansion of the general determinant of a matrix to be equivalent to $n$ !, where $\mathrm{n}$ represented the number of columns (or rows) of the matrix (Metzler, 1960). Indeed, the same quantity re-appears in the results of this study, but the calculation here is done using the first row of a matrix.

Since then, interest in general expressions or relations between determinants and the number of minors of various orders and elements contained in them has faded. More recently though some references have begun to appear in mathematical literature; three examples of such are (Malek, 2011; Wilde et al., 2010; Jones, 2011).

Whilst the results of the studies discussed above provide much insight into properties of general determinants, independent principal minors and the relations between them, little attention was paid to finding expressions for the total number of minors (of various orders) and elements in a general determinant (irrespective of whether the minors and elements are principal or independent). If the determinant of a matrix is to be examined with respect to the number of minors and elements contained in the determinant, then these

Corresponding Author: Gurudeo Anand Tularam, Department of Mathematics and Statistics,

Science Environment Engineering and Technology (ENV), Griffith University, Brisbane Australia 
results pertain only to the case when the minors and/or elements in question are independent and situated with principality in the associated matrix. In an attempt to fill this gap, the authors present expressions in this study for the total number of minors (of various orders) and elements in a general determinant, irrespective of the independence, principality and distinctness of such minors and elements. The proofs of the same are also included.

\section{Method, analysis and results:}

Notation and terminology: Herein, the term uniqueness refers to the qualities of distinctness, independence and principality of a minor or element. That is, when counting the various minors and elements that are present in the complete expansion of the general determinant, each minor and element is counted as many times as it appears.

$\mathrm{A}_{\mathrm{n}}$ denotes a square matrix of dimensions $\mathrm{n} \times \mathrm{n}$ :

- $\quad r: r \in\{2,3,4, \ldots, n\}$, denotes a minor of order $r$ of the matrix, $A_{n}$. Here order is defined as the size of the respective minor. As such, the statement $r=n$ is to interpreted as the general determinant of the matrix, $A_{n}$

- $\quad \mathrm{N}(\mathrm{n}, \mathrm{r}): \mathrm{r} \in\{2,3,4, . ., \mathrm{n}\}, \mathrm{n} \in\{2,3,4, \ldots\}$ denotes the number of minors of order $r$, irrespective of uniqueness of such minors, that appear in the general determinant of $A_{n}$

- $\mathrm{a}_{\mathrm{ij}}$ : $\mathrm{i} \in\{1,2,3, . ., \mathrm{n}\}, \mathrm{j} \in\{1,2,3, . . \mathrm{n}\}$ denotes an element of $A_{n}$ situated in the $i^{\text {th }}$ row and $j$ th column of $A_{n}$

- $\quad \mathrm{N}\left(\mathrm{a}_{\mathrm{ij}}\right)$ denotes the number of elements, irrespective of uniqueness of each element, that appear in the general determinant of $A_{n}$

Beginning with the case when $\mathrm{n}=2$ the determinant is then given by:

$$
\operatorname{det}\left(\mathbf{A}_{2}\right)=\left|\begin{array}{ll}
\mathrm{a}_{11} & \mathrm{a}_{12} \\
\mathrm{a}_{21} & \mathrm{a}_{22}
\end{array}\right|=\mathrm{a}_{11} \mathrm{a}_{22}-\mathrm{a}_{12} \mathrm{a}_{21},
$$

and so:

- $\quad \mathrm{N}\left(\mathrm{a}_{\mathrm{ij}}\right)=4$

- $\mathrm{N}(2,2)=1$

And when we consider the case $\mathrm{n}=3$ determinant is:

$$
\begin{aligned}
& \operatorname{det}\left(\mathbf{A}_{3}\right)=\left|\begin{array}{lll}
a_{11} & a_{12} & a_{13} \\
a_{21} & a_{22} & a_{23} \\
a_{31} & a_{32} & a_{33}
\end{array}\right|=a_{11}\left(a_{22} a_{33}-a_{23} a_{32}\right) \\
& -a_{12}\left(a_{21} a_{33}-a_{23} a_{31}\right)+a_{13}\left(a_{21} a_{32}-a_{22} a_{31}\right)
\end{aligned}
$$

The right hand side of the above determinant expression may be re-written as:

$$
\begin{aligned}
& a_{11} a_{22} a_{33}-a_{11} a_{23} a_{32}-a_{12} a_{21} a_{33}+ \\
& a_{12} a_{23} a_{31}+a_{13} a_{21} a_{32}-a_{13} a_{22} a_{31}
\end{aligned}
$$

And if uniqueness of all minors and elements in the expansion is not considered, then:

- $\mathrm{N}\left(\mathrm{a}_{\mathrm{ij}}\right)=18$

- $\mathrm{N}(3,2)=3$

- $\mathrm{N}(3,3)=1$

Similarly when we consider the case of $n=4$, the determinant is:

$$
\begin{aligned}
& \operatorname{det}\left(A_{4}\right)=\left|\begin{array}{llll}
a_{11} & a_{12} & a_{13} & a_{14} \\
a_{21} & a_{22} & a_{23} & a_{24} \\
a_{31} & a_{32} & a_{33} & a_{34} \\
a_{41} & a_{42} & a_{43} & a_{44}
\end{array}\right| \\
& =a_{11}\left[a_{22}\left(a_{33} a_{44}-a_{34} a_{43}\right)-a_{23}\right. \\
& \left.\left(a_{32} a_{44}-a_{34} a_{42}\right)+a_{24}\left(a_{32} a_{43}-a_{33} a_{42}\right)\right] \\
& -a_{12}\left[a_{21}\left(a_{33} a_{44}-a_{34} a_{43}\right)-a_{23}\left(a_{31} a_{44}-a_{34} a_{41}\right)\right. \\
& \left.+a_{24}\left(a_{31} a_{43}-a_{33} a_{41}\right)\right] \\
& +a_{13}\left[a_{21}\left(a_{32} a_{44}-a_{34} a_{42}\right)-a_{22}\left(a_{31} a_{44}-a_{34} a_{41}\right)\right. \\
& \left.+a_{24}\left(a_{31} a_{42}-a_{32} a_{41}\right)\right] \\
& -a_{14}\left[a_{21}\left(a_{32} a_{43}-a_{33} a_{42}\right)-a_{22}\left(a_{31} a_{43}-a_{33} a_{41}\right)\right. \\
& \left.+a_{23}\left(a_{31} a_{42}-a_{32} a_{41}\right)\right]
\end{aligned}
$$

Rewriting the above expansion similarly as in the $3 \times 3$ case and disregarding uniqueness of all minors and elements the following minors are noted:

- $\mathrm{N}\left(\mathrm{a}_{\mathrm{ij}}\right)=96$

- $\mathrm{N}(4,2)=12$

- $N(4,3)=4$

- $\mathrm{N}(4,4)=1$

In the same manner, the following results are obtained when, $\mathrm{n}=5$ :

- $\mathrm{N}\left(\mathrm{a}_{\mathrm{ij}}\right)=600$

- $\mathrm{N}(5,2)=60$

- $\mathrm{N}(5,3)=20$

- $\mathrm{N}(5,4)=5$

- $\mathrm{N}(5,5)=1$

Main results: From the above analysis, an expression for the number of minors of order, $r \geq 2$, irrespective of 
uniqueness, that are present in the expansion of the general determinant of $A_{n}$ is Eq. 1:

$$
\mathrm{N}(\mathrm{n}, \mathrm{r})=\frac{\mathrm{n} !}{\mathrm{r} !}, \mathrm{r} \in\{2,3,4, . ., \mathrm{n}\}, \mathrm{n} \in\{2,3,4, \ldots\}, \mathrm{r} \leq \mathrm{n}
$$

The expression for the number of elements, irrespective of uniqueness, that are present in the complete expansion of the general determinant of $A_{n}$ is the given by Eq. 2 :

$$
N\left(a_{i j}\right)=n^{2}(n-1) !, n \in\{2,3,4, \ldots\}
$$

\section{Proofs:}

Case 1:

$$
r \in\{2,3,4, . ., n\}, n \in\{2,3,4, \ldots\}, r \leq n
$$

Sub-Case 1.1: $\mathbf{r}<\mathbf{n}$ : Firstly, we note that this sub-case presents restrictions on $r$ and $n$, such that $r \geq 2$ and $n \geq 3$.

Then the following is observed.

The general determinant of a square matrix $A_{n}$ must contain $n$ minors of order $r=n-1$ :

$$
\therefore \mathrm{N}(\mathrm{n}, \mathrm{n}-1)=\mathrm{n}, \mathrm{r}=\mathrm{n}-1
$$

Likewise, a minor of order $r=n-1$ that is in the general determinant of $A_{n}$ must contain $n-1$ minors of order, $n-2$, each of which is also in the general determinant of $A_{n}$ and thus it follows that:

$$
\begin{aligned}
& N(n, n-2)=n(n-1), r=n-2 \\
& N(n, n-3)=n(n-1)(n-2), r=n-3 \\
& N(n, n-4)=n(n-1)(n-2)(n-3), r=n-4 \ldots . \\
& \text { So } \forall n \in\{3,4,5, \ldots\} \text { and } \\
& \forall r \in\{n-1, n-2, n-3, . ., 2\}: 1<r<n, \\
& N(n, r)=\prod_{k=1}^{(n-r)}(r+k)=(r+1)(r+2)(r+3), . ., \times n \\
& =n(n-1)(n-2)(n-3), . ., \times(r+1) \\
& =\frac{n !}{r !}, r<n, n \in\{3,4,5, \ldots\}, r \in\{2,3,4, . .\}
\end{aligned}
$$

Sub-Case 1.2: $\mathbf{r}=\mathbf{n}$ : As noted earlier, this gives the general determinant of $A_{n}$ and as such, this sub-case is trivial.

Proving the converse of the above is present in the following.

Let us suppose the case when $r \neq n$. Then $r<n$ such that, $\mathrm{n} \in\{3,4,5, \ldots\}$ and $\mathrm{r} \in\{2,3,4, \ldots\}$. Thus

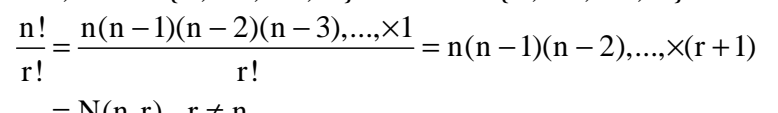

Now let us suppose $r=n$. Again, this gives the general determinant of $A_{n}$ and as such, this sub-case is trivial.

Thus:

$$
\begin{aligned}
& \forall \mathrm{r} \in\{2,3,4, . .\} \text { and } \forall \mathrm{n} \in\{2,3,4, \ldots\} \\
& \text { with } \mathrm{r} \leq \mathrm{n}, \mathrm{N}(\mathrm{n}, \mathrm{r}) \Leftrightarrow \frac{\mathrm{n} !}{\mathrm{r} !}
\end{aligned}
$$

Case 2:

$$
\begin{aligned}
& \mathrm{N}\left(\mathrm{a}_{\mathrm{ij}}\right)=\mathrm{n}^{2}(\mathrm{n}-1) !, \\
& \mathrm{n} \in\{2,3,4, \ldots\}
\end{aligned}
$$

Firstly, it is observed that for, $n \geq 2$, any minor of order $r=2$ that appears in the complete expansion of the general determinant of $A_{n}$ will be multiplied by $n-2$ elements $\quad a_{i j}: \quad i \in\{1,2,3, . ., n\}, \quad j \in\{1,2,3, . . n\}$ corresponding to the $\mathrm{n}-2$ elements lying at the intersections of the rows and columns that must be struck out in order to isolate that minor. Upon multiplication, each of these n-2 elements contributes one element to each of the additive terms in each of these minors. Consequently, when a minor of order $r=2$ is multiplied by these $n-2$ elements, the number of elements contributed to the complete expansion of the determinant by that multiplication is equivalent to $(n-2)+2+(n-2)+2=2 n$.

Using the result proved in Case 1 earlier, the number of minors of order $r=2$ irrespective of uniqueness in the general determinant of $A_{n}$ is given by, $\mathrm{N}(\mathrm{n}, 2)=\frac{\mathrm{n} !}{2 !}=\frac{\mathrm{n} !}{2}$. It then follows that for $\mathrm{n} \geq 2$, the total number of elements, irrespective of uniqueness, in the complete expansion of the general determinant is given by:

$$
\begin{aligned}
N\left(a_{i j}\right) & =N(n, 2) \times(2 n)=\frac{n !}{2 !}(2 n)=n \times n ! \\
& =n^{2}(n-1) ! n \in\{2,3,4, \ldots\}
\end{aligned}
$$

Proving the converse of the above then follows:

$$
\begin{aligned}
\mathrm{n}^{2}(\mathrm{n}-1) ! & =\mathrm{n} \times \mathrm{n} \times(\mathrm{n}-1) \times(\mathrm{n}-2) \times(\mathrm{n}-3) \times . . \times 1 \\
& =\mathrm{n} \times \mathrm{n} !==\frac{\mathrm{n} !}{2 !}(2 \mathrm{n})=\mathrm{N}(\mathrm{n}, 2) \times(2 \mathrm{n})=\mathrm{N}\left(\mathrm{a}_{\mathrm{ij}}\right)
\end{aligned}
$$

Thus:

$$
\forall \mathrm{n} \in\{1,2,3, \ldots\}, \mathrm{N}\left(\mathrm{a}_{\mathrm{ij}}\right) \Leftrightarrow \mathrm{n}^{2}(\mathrm{n}-1) !
$$




\section{CONCLUSION}

The results of this study present, with proof, expressions for the total number of minors of various orders and elements in a general determinant, irrespective of the independence, principality and distinctness of such minors and elements. The mathematics educational literature, particularly in the field of Linear Algebra, could employ these results as a contribution to the known properties of determinants. The results also indicate scope for further theoretical study of this property with applications in applied mathematics and the physical and computer sciences. A geometric interpretation of the results or a method for the calculation of general determinants based on these results would be logical subsequent theoretical studies, both having applications that would extend to the physical and computer sciences.

\section{REFERENCES}

Jones, J., 2011. The determinant of a square matrix.

Malek, M., 2011. Linear algebra: Characteristic polynomial.

Metzler, W.H., 1960. A Treatise on the Theory of Determinants. 1st Edn., Dover Publications Inc., New York, pp: 766.

Muir, T., 2008. The Theory of Determinants in the Historical Order of Development. 1st Edn., BiblioBazaar, ISBN-10: 0554808730, pp: 222.

Stouffer, E.B., 1928. Expressions for the general determinant in terms of its principal minors. Am. Math. Monthly, 35: 18-21.

Wilde, J., T. Revised Suzuki and I. Tecu, 2010. Linear Algebra II: Quadratic forms and definiteness. 